\title{
Pancreatic islet and progenitor cell surface markers with cell sorting potential
}

\author{
J. Hald • T. Galbo • C. Rescan • L. Radzikowski • A. E. Sprinkel • H. Heimberg • \\ J. Ahnfelt-Rønne • J. Jensen - R. Scharfmann • G. Gradwohl • K. H. Kaestner • \\ C. Stoeckert Jr • J. N. Jensen • O. D. Madsen
}

Received: 17 March 2011 / Accepted: 12 July 2011 /Published online: 23 September 2011

(C) Springer-Verlag 2011

\begin{abstract}
Aims/hypothesis The aim of the study was to identify surface bio-markers and corresponding antibody tools that can be used for the imaging and immunoisolation of the pancreatic beta cell and its progenitors. This may prove essential to obtain therapeutic grade human beta cells via stem cell differentiation.

Methods Using bioinformatics-driven data mining, we generated a gene list encoding putative plasma membrane proteins specifically expressed at distinct stages of the developing pancreas and islet beta cells. In situ hybrid-
\end{abstract}

Electronic supplementary material The online version of this article (doi:10.1007/s00125-011-2295-1) contains peer-reviewed but unedited supplementary material, which is available to authorised users.

J. Hald · T. Galbo · C. Rescan · J. Ahnfelt-Rønne · J. N. Jensen •

O. D. Madsen $(\square)$

Department of Beta-Cell Regeneration,

Hagedorn Research Institute,

Niels Steensens Vej 1,

2820 Gentofte, Denmark

e-mail: odm@hagedorn.dk

J. Hald · T. Galbo · L. Radzikowski • A. E. Sprinkel •

O. D. Madsen

Antibody Core, Beta Cell Biology Consortium

URL: www.betacell.org

L. Radzikowski · A. E. Sprinkel

Department of Antibody Technology, Novo Nordisk, Måløv, Denmark

H. Heimberg

Diabetes Research Center, Vrije Universiteit Brussel, Brussels, Belgium isation and immunohistochemistry were used to further prioritise and identify candidates.

Results In the developing pancreas seizure related 6 homologue like (SEZ6L2), low density lipoprotein receptor-related protein 11 (LRP11), dispatched homologue 2 (Drosophila) (DISP2) and solute carrier family 30 (zinc transporter), member 8 (SLC30A8) were found to be expressed in early islet cells, whereas discoidin domain receptor tyrosine kinase 1 (DDR1) and delta/notch-like EGF repeat containing (DNER) were expressed in early pancreatic progenitors. The expression pattern of DDR1
J. Jensen

Department of Stem Cell Biology and Regenerative Medicine,

Cleveland Clinic Lerner Research Institute,

Cleveland, OH, USA

R. Scharfmann

Inserm U845, Research Center Growth and Signalling, Université

Paris Descartes, Hôpital Necker,

Paris, France

G. Gradwohl

Institut de Génétique et de Biologie Moléculaire et Cellulaire, Inserm U964, CNRS UMR 7104, Université de Strasbourg, Illkirch, France

K. H. Kaestner

Department of Genetics, University of Pennsylvania,

Philadelphia, PA, USA

C. Stoeckert Jr

Penn Center for Bioinformatics and Department of Genetics,

University of Pennsylvania,

Philadelphia, PA, USA 
overlaps with the early pancreatic and duodenal homeobox 1 (PDX1) $^{+} / \mathrm{NK} 6$ homeobox 1 (NKX6-1) $^{+}$multipotent progenitor cells from embryonic day 11, whereas DNER expression in part overlaps with neurogenin 3 (NEUROG3 $)^{+}$cells. In the adult pancreas SEZ6L2, LRP11, DISP2 and SLC30A8, but also FXYD domain containing ion transport regulator 2 (FXYD2), tetraspanin 7 (TSPAN7) and transmembrane protein 27 (TMEM27), retain an isletspecific expression, whereas DDR1 is undetectable. In contrast, DNER is expressed at low levels in peripheral mouse and human islet cells. Re-expression of DDR1 and upregulation of DNER is observed in duct-ligated pancreas. Antibodies to DNER and DISP2 have been successfully used in cell sorting.

Conclusions/interpretation Extracellular epitopes of SEZ6L2, LRP11, DISP2, DDR1 and DNER have been identified as useful tags by applying specific antibodies to visualise pancreatic cell types at specific stages of development. Furthermore, antibodies recognising DISP2 and DNER are suitable for FACS-mediated cell purification.

Keywords Beta cell · DDR1 - DNER - DISP2 - LRP11 . Pancreas $\cdot$ Progenitor $\cdot$ SEZ6L2 $\cdot$ Surface bio-marker

$\begin{array}{ll}\text { Abbreviations } & \\ \text { DDR1 } & \text { Discoidin domain receptor tyrosine kinase 1 } \\ \text { DISP2 } & \text { Dispatched homologue 2 (Drosophila) } \\ \text { DNER } & \text { Delta/notch-like EGF repeat containing } \\ \text { e18.5 } & \text { Embryonic day 18.5 in the mouse } \\ \text { EPCon } & \text { Endocrine pancreas consortium } \\ \text { EST } & \text { Expressed sequence tag } \\ \text { FXYD2 } & \text { FXYD domain containing ion transport } \\ & \text { regulator 2 } \\ \text { HEK-F } & \text { Freestyle suspension human embryonic } \\ & \text { kidney 293 cells } \\ \text { HEPACAM2 } & \text { HEPACAM family member 2 } \\ \text { hESCs } & \text { Human embryonic stem cells } \\ \text { IHC } & \text { Immunohistochemistry } \\ \text { ISH } & \text { In situ hybridisation } \\ \text { KLH } & \text { Keyhole limpet haemocyanin } \\ \text { LRP11 } & \text { Low density lipoprotein receptor-related } \\ & \text { protein 11 } \\ \text { NEUROG3 } & \text { Neurogenin 3 } \\ \text { NKX6.1 } & \text { NK6 homeobox 1 } \\ \text { PDL } & \text { Pancreatic duct ligation } \\ \text { PDX1 } & \text { Pancreatic and duodenal homeobox 1 } \\ \text { PROM1 } & \text { Prominin 1 } \\ \text { PTF1A } & \text { Pancreas specific transcription factor, 1a } \\ \text { SEZ6L2 } & \text { Seizure related 6 homologue like } \\ \text { SLC30A8 } & \text { Solute carrier family 30 (zinc transporter), } \\ & \text { member } 8 \\ \text { TMEM27 } & \text { Transmembrane protein 27 } \\ \text { TSPAN7 } & \text { Tetraspanin } 7\end{array}$

WG

YFP

Weeks of gestation

Yellow fluorescent protein

\section{Introduction}

As diabetes is caused by reduction of functional beta cell mass (absolute vs relative in type 1 diabetes vs type 2 diabetes [1-3]) much emphasis is focused on beta cell regeneration and replacement. Neogenesis of functional beta cells occurs in mice following partial pancreatic duct ligation (PDL) [4]. Proof-of-principle transplantation studies in type 1 diabetes patients have demonstrated that reconstitution of functional beta cell mass can re-establish normoglycaemia $[5,6]$. Limited access to human donor islet material has stimulated activities with the aim of generating therapeutic beta cells from pluripotent human embryonic stem cells (hESCs) and induced pluripotent stem cells [7-11]. hESCs have currently been differentiated towards heterogeneous cultures containing beta cell-like cells that are often multihormonal $[8,9]$.

Antibody-mediated cell sorting has been instrumental in dissecting the complexity of the haematopoietic system [12]. An expanding collection of surface marker antibodies have been generated towards mature pancreatic cells through cellular immunisation [13], autoimmune hybridoma formation $[14,15]$, islet-specific panning of phagelibraries [16-18] and by bioinformatics approaches [19]. Stage-specific surface markers may serve as purification tags to better characterise and purify relevant cell populations during pancreatic differentiation, regeneration and directed differentiation in vitro.

Chemokine (C-X-C motif) receptor 4 (CXCR4) has been identified as a definitive endoderm-specific marker during differentiation of ESC cells towards endoderm SRY (sex determining region Y)-box 17-positive $\left(\mathrm{SOX} 17^{+}\right)$[20]. It has been applied with success by Cai et al. [21] where the $\mathrm{CXCR}^{+}{ }^{+}$vs $\mathrm{CXCR}^{-}$sorted cells yielded up to $95 \%$ vs $<10 \%$ pancreatic and duodenal homeobox 1-positive $\left(\mathrm{PDX}^{+}\right)$cell population upon further differentiation [21].

The cell surface antigen CD133 (also known as prominin 1 [PROM1]) has been identified as a marker of a cell population that may contain neurogenin 3 (NEUROG3)positive pancreatic endocrine progenitor cells [22]. Enrichment of NEUROG3-positive cells in this PROM1-positive fraction was, however, only calculated to be 1.7-fold and most cells isolated by PROM1 were carboxypeptidase A (CPA1)-positive.

Surface markers for mature islet cell types have been published and include FXYD domain containing ion transport regulator 2 (FXYD2) [19], tetraspanin 7 (TSPAN7) [23], transmembrane protein 27 (TMEM27) 
[24-27] and delta- [14], alpha- [18, 28] and beta cellspecific antibodies [18], as well as pan-islet [28] antibodies recognising unknown structures.

In this study we present a bioinformatics approach combined with in situ hybridisation/immunohistochemistry (IHC) screen allowing the identification of novel surface bio-markers of endocrine progenitors (discoidin domain receptor tyrosine kinase 1 [DDR1] and delta/notch-like EGF repeat containing [DNER]) and mature islet of Langerhans cells (dispatched homologue 2 (Drosophila) [DISP2], seizure related 6 homologue like [SEZ6L2], low density lipoprotein receptor-related protein 11 [LRP11] and HEPACAM family member 2 [HEPACAM2]). We also confirmed TSPAN7 and TMEM27 to be on our list. Furthermore, we show that DISP2 and DNER can serve as tags for cell purification using specific monoclonal and polyclonal antibodies.

\section{Methods}

Generation of gene lists Genes on the Neurog3-knockout list (array list 1 in electronic supplementray material [ESM] Table 1) were based on array data from mouse pancreas isolated from CD1 Neurog $3^{-/}$and wild-type embryos at embryonic day 18.5 (e18.5) [29]. On this list we expect to find genes characterising mature endocrine cells.

Genes on the Neurog3-yellow fluorescent protein (YFP) list (array list 2 in ESM Table 1) were based on array data from e15.5 Neurog $3^{\mathrm{eYFP} / \mathrm{t}}$-positive and -negative FACSsorted pancreatic cells [30]. On this list we could possibly identify genes regulated similarly to Neurog3 in the endocrine progenitors.

The unbiased list (array list 3 in ESM Table 1) was generated by identifying assemblies in the Database of Transcribed Sequences (DoTS) contained in the Endocrine Pancreas Consortium (EPCon) libraries and then sorting for relevant surface proteins by bioinformatics. This list would be expected to contain any pancreatic surface marker gene. For further details, see ESM Detailed research design and methods.

Prioritisation of candidates Candidates were prioritised according to: existence of human orthologues, transmembrane properties, plasma membrane localisation and degree of differential expression in the whole body. For further details, see ESM Detailed research design and methods.

In situ hybridisation Probes of approximately $500 \mathrm{bp}$ were designed and cloned in pCR4-TOPO TA cloning vector from e15.5 mouse gut RNA. Digoxigenin (DIG)-labelled RNA probes were generated and hybridisation was carried out as described [31]. Briefly, slides were thawed at room temperature, probe added at $1 \mathrm{ng} / \mu \mathrm{l}$ overnight at $65^{\circ} \mathrm{C}$, washed and anti-DIG antibody was added overnight at room temperature. In situ signal was developed with 5-bromo-4chloro-3'-indolyphosphate/ nitro-blue tetrazolium (BCIP/ $\mathrm{NBT}$ ) in $\mathrm{NaCl}$, Tris-HCl, $\mathrm{MgCl}$, Tween-20 (NTMT) buffer.

Peptide selection for immunisation Candidates were evaluated towards post-translational modifications and with respect to linear B cell epitopes by using CBS Prediction Servers (www.cbs.dtu.dk/services/). Furthermore, potential peptide areas were compared between mouse/human species to identify conserved versus species-specific epitopes. Using BLASTp, selected peptides with potential crossreactivity were eliminated. Finally, a cysteine was added to facilitate subsequent coupling to a carrier-protein keyhole limpet haemocyanin (KLH). Selected peptides mapping to the extra-cellular side were synthesised (Schafer-N, Copenhagen, Denmark) for immunisation.

KLH coupling Peptides were conjugated to KLH using Imject Maleimide Activated mcKLH (mcKLH) Kit (Pierce \#77611; Thermo Fisher Scientific, Rockford, IL, USA) according to the manufacturer's instructions. The conjugates were purified by dialysis in PBS overnight.

Immunisations and antiserum generation The KLHconjugated peptide was injected into RBF (Robertsonian POSF) mice or into rabbits. For further details, see ESM Detailed research design and methods.

Production of monoclonal antibodies A mouse with hightitred serum was chosen for production of monoclonal DISP2 antibodies and was boosted intravenously with $10 \mu \mathrm{g}$ antigen. Spleen cells were fused and hybridoma supernatant fractions were screened by indirect ELISA for specific binding to the immunising antigen. The secondary screening of positive hits from the primary screen was performed by IHC staining of sections of adult mouse pancreas.

Tissue preparation Whole mouse embryos, dissected guts and adult pancreas were isolated, fixed in $4 \%$ paraformaldehyde (PFA), embedded in optimum cutting temperature Tissue-Tech (OCT) and sectioned as frozen $(10 \mu \mathrm{m}$ section) or stored in methanol. Tissue from one human adult non-pathological pancreas from a heart-beating, cadaveric, non-diabetic donor was procured at a European hospital associated with the Eurotransplant Foundation (Leiden, the Netherlands) and with the beta cell bank of the JDRF Center for Beta Cell Therapy of Diabetes, as approved by the ethical committee of the Free University of Brussels ('Commissie Medische Ethiek-VUB', reference \#2002/VS). 
Cell culture AlphaTC cells were grown in DMEM low (1,000 mg/l D-glucose, sodium pyruvate), $10 \% \mathrm{FCS}$, and $100 \mathrm{U}$ of penicillin and $50-100 \mu \mathrm{g}$ of streptomycin. Freestyle suspension human embryonic kidney 293 (HEK-F) cells were grown in freestyle 293 medium (Gibco, \#12338; Invitrogen $\mathrm{A} / \mathrm{S}$, Hellerup, Denmark), with $100 \mathrm{U}$ of penicillin and $100 \mu \mathrm{g}$ of streptomycin added. AlphaTC and HEK-F cells were harvested with cell dissociation solution C5789-100 M (Sigma-Aldrich, Brøndby, Denmark) or 0.05\% Trypsin-EDTA (Invitrogen, Hellerup, Denmark), washed and fixed for $1 \mathrm{~h}$ at room temperature, subsequently washed in PBS and stored at $+5^{\circ} \mathrm{C}$ until use.

Immunohistochemistry IHC on sections and data collection was carried out as described previously [32]. Whole mount IHC and data collection were performed essentially as described previously [33]. Primary antibodies were used and detected as listed in ESM Table 2.

In vitro differentiation of human embryonic pancreas Human fetal pancreases from tissue fragments were obtained immediately after elective termination of pregnancy as previously described [34, 35]. For culture, pancreases were laid on Millicell culture plate inserts (Millipore, Billerica, MA, USA) in $60 \mathrm{~mm}$ sterile Petri dishes containing $5 \mathrm{ml}$ of RPMI 1640 (Invitrogen) supplemented with penicillin $(100 \mathrm{U} / \mathrm{ml})$, streptomycin $(100 \mu \mathrm{g} / \mathrm{ml})$, Hepes $(10 \mathrm{mmol} / \mathrm{l})$, L-glutamine $(2 \mathrm{mmol} / \mathrm{l})$, non-essential amino acids $(1 \times$, Gibco) and $10 \%$ heat-inactivated calf serum (Hyclone, Logan, UT, USA). Under such culture conditions, the pancreases grew at the air/medium interface as described for rodent pancreases [36, 37]. Cultures were maintained at $37^{\circ} \mathrm{C}$ in humidified $95 \%$ air $/ 5 \% \mathrm{CO}_{2}$. The medium was changed every other day.

$P D L$ The pancreatic duct of 8-week-old Balb/C mice was ligated as described [4]. Ligated and sham-operated animals were killed at day 7 after surgery and preserved for IHC as above.

Cytospin Preparation of harvested alphaTC and HEK-F cells for immunocytochemistry was done by spinning cells on to a microscope slide in a StatSpin Cytofuge2 for $4 \mathrm{~min}$ at $850 \mathrm{rpm}(40 \mathrm{~g})$. Cells were stained like IHC tissue sections; however, peroxidase quenching by $\mathrm{H}_{2} \mathrm{O}_{2}$ block was omitted.

Flow cytometry Cells were blocked in 5\%, 0.5\% Tris$\mathrm{NaCl}$-blocking reagent buffer (TNB) (PerkinElmer, Skovlunde, Denmark) and 5\% donkey serum at room temperature for $1 \mathrm{~h}$. Primary antibody was added (mouse immunoglobulin [Ig]G1 anti-DISP2, goat IgG anti-DNER, isotype mouse $\mathrm{IgG1}$ and non-immune goat $\mathrm{IgG}$ ) and incubated for $2 \mathrm{~h}$ at room temperature with occasional gentle mixing. After washing in PBS, cells were incubated with secondary antibody for $1 \mathrm{~h}$. Finally, cells were washed in PBS. Stained cells were analysed on a FACSAria flow cytometer (BD Biosciences [Becton, Dickinson, Brøndby, Denmark]) in at least three independent experiments. Graphs were made in FSC Express (De Novo Software, Los Angeles, CA, USA).

Image handling The optical stacks of the whole mount stainings were trimmed and equidistant subsets of sections were used to generate visually informative projections. For DNER/paired box 6 (PAX6), DNER/NEUROG3, DNER/ PDX1 and DNER/insulin/glucagon every second, third, third and eighth optical section was used respectively. Double and triple overlays were done in LSM Image Browser (http://www.zeiss.de) or Adobe Photoshop (ATEA, Ballerup, Denmark).

\section{Results}

Bioinformatics ranking of candidates To identify biosurface markers selectively expressed in the islet of Langerhans and in the endocrine progenitors we took advantage of Neurog3 null [29] as well as of Neurog3YFP [38] array data. An additional unbiased list of potential pancreas bio-surface markers (the EpconDB list) was generated as a shared resource within the Beta Cell Biology Consortium.

Based on multiple criteria (human orthologue, transmembrane protein, plasma membrane localised protein, none ubiquitously expression, degree of pancreas-restricted expression) genes from these three input lists were prioritised in silico (Fig. 1). (All genes are listed in ESM Table 1.) The prioritisation resulted in a list of 151 candidate genes encoding pancreatic transmembrane proteins to be further analysed by the in situ hybridisation (ISH) and IHC screens.

ISH and IHC screen The 151 candidates were screened by ISH (e15.5 mouse pancreas and mining Genepaint database) to evaluate the spatial expression pattern. We scored the expression pattern into the following groups (Fig. 1): (1) ubiquitous; (2) tissue-specific, but non-pancreatic. Genes expressed in the pancreas ( 3 through 8 ) were scored as (3) broad; (4) mesenchymal; (5) acinar; (6) 'Nkx6.1-like'; (7) 'Neurog3-like'; and (8) 'Endocrine-like'. A total of 14 genes were scored within the favourable range of ' $6-8$ '; and Ddr1 and Disp2 are examples of this (Fig. 2a and b, and Fig. 1). Another 137 candidates were found to have an ISH signal that excluded them from further investigations. Kcnkl and Epha7 are examples of this (Fig. 2c and d, and 


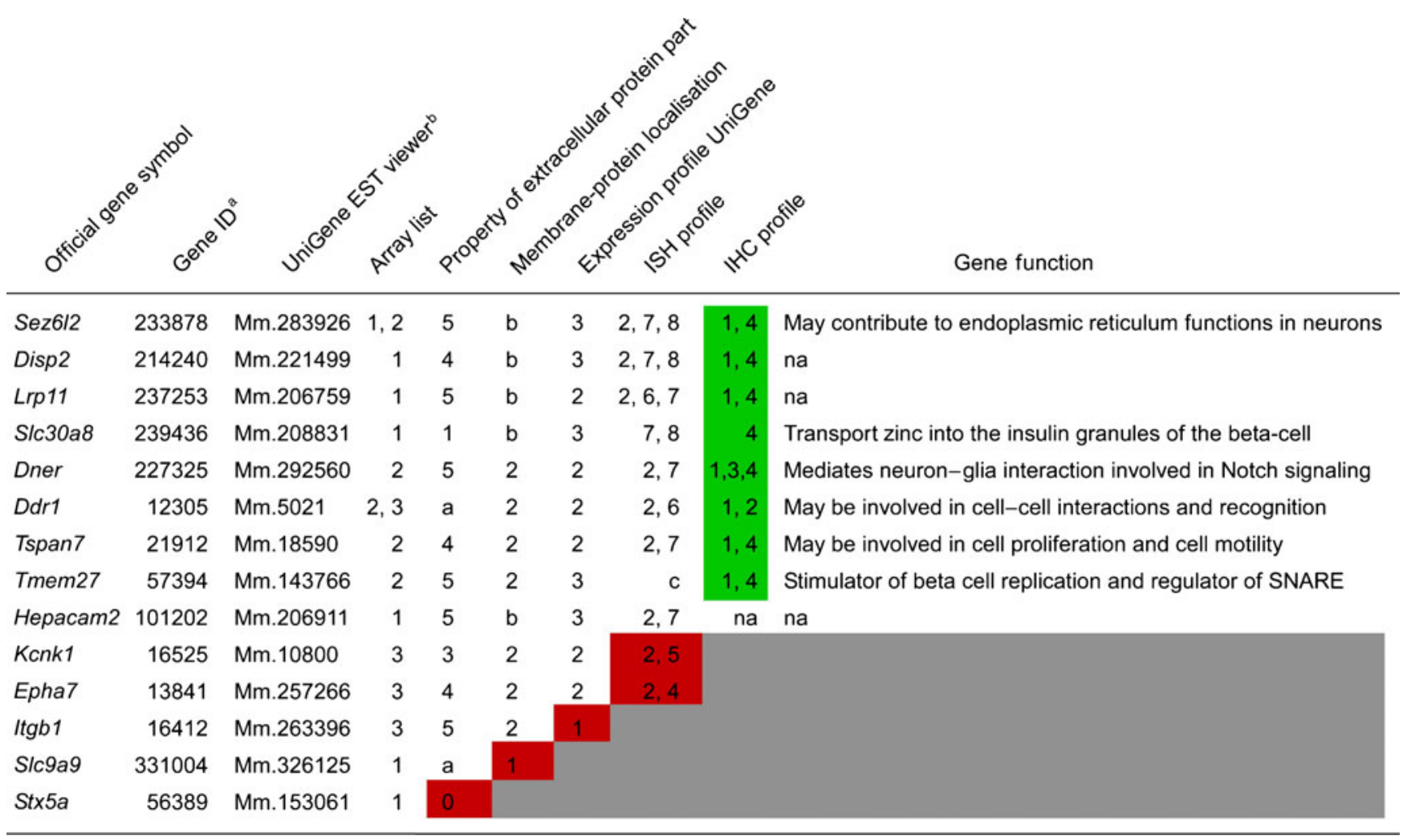

Fig. 1 Prioritised list of candidates. Genes in the screen are listed by their official gene symbol. For each gene hyperlinks are listed to NCBI gene ID and the UniGene expressed sequence tag (EST) Profile Viewer. Numbers in the 'Array list' correspond to the particular gene list (see ESM Detailed research design and methods) on which the gene was identified: '1' Neurog3 null; '2' Neurog3-YFP; '3' EPCon. 'Property of extracellular protein part' and transmembrane (TM) topology was scored as follows: ' 0 ' Less than 5 consecutive extracellular aa; ' 1 ' Less than 20 consecutive extracellular aa; ' 2 ' 20-50 consecutive extracellular aa; ' 3 ' 50-100 consecutive extracellular aa; '4' More than 100 consecutive extracellular aa with multi TM spans; ' 5 ' More than 100 consecutive extracellular aa single TM; 'a' Prediction of inside/outside orientation of protein not conclusive. 'Membrane-protein localisation' was evaluated and scored as follows: ' 1 ' Protein is not on the cell surface; ' 2 ' Protein is exposed to the cell surface (part of the plasma membrane); ' $b$ ' Gene is not listed in IPA (Ingenuity Pathway Analysis). 'Expression profile UniGene' evaluates the degree of tissue-specific expression (in mice) of a gene using UniGene EST Profile Viewer: ' 1 ' Broad expression; '2' Differential expression; ' 3 ' Highly differentially expression. At this stage, a total

Fig. 1). Prioritisation and ranking (Fig. 1) was performed through the above ISH screen and by IHC using pre-existing or own production of antibodies in order to eventually identify extracellular bio-surface marker epitopes.

The detailed IHC screen identified DDR1 and DNER as potential endocrine progenitor bio-surface markers; DISP2, LRP11, SEZ6L2 and solute carrier family 30 (zinc transporter), member 8 (SLC30A8) as potential endocrine markers and HEPACAM2 as a potential beta cell selective marker (HEPACAM2 to be described elsewhere by of 197 genes were reduced to 151 genes to be evaluated further by ISH on mouse embryo sections (in house or GenePaint). 'ISH profile' was scored as follows: '1' Broad expression/background; '2' Specific non-pancreas expression. Genes expressed within the pancreas were scored as ' 3 ' Broad; '4' Mesenchymal; '5' Acinar; '6' Nkx6.1-like; '7' Neurog3-like; '8' Endocrine-like; 'c' Data not conclusive/data not available. The ISH screen eliminated 65 genes to allow focus on analysing the protein expression of 86 top-candidates by IHC. The pancreatic 'IHC profile' was scored as follows: ' 1 ' Absent ' 2 ' NKX6.1-like; '3' NEUROG3-like; '4' Hormone-positive cells. 'Gene function' was extracted from UniProt. 'na' no data available. Entries are shaded red if genes were discontinued based on the criteria of the respective column. Grey shading indicates that the gene has not been evaluated. Green shading indicates that genes have passed IHC criteria. ${ }^{a}$ URL to access gene online at NCBI: www.ncbi.nlm.nih. gov/sites/entrez? $\mathrm{db}=$ gene\&cmd=search\&term $=$ and type the "Gene ID" number in the search box. ' URL to access the EST viewer profile at NCBI: www.ncbi.nlm.nih.gov/UniGene/ESTProfileViewer.cgi?uglist= EST-ID "EST-ID"=UniGene EST viewer entry

J. Jensen) (Fig. 1). So far, we have been unable to make monoclonal antibodies to the small extracellular domains of SLC30A8 (polyclonal antisera are commercially available, Mellitech [Grenoble, France], PZ8 and RZ8). Here, we describe the expression patterns of the five novel surface proteins in more detail: DDR1 (GeneID: 25678) is a singlespan transmembrane receptor tyrosine kinase with 414 extracellular amino acids (aa). DDR1 is activated by ectodomain binding to fibrillar collagen [39]. DDR1ectodomain shedding has been described [40]. $D d r 1^{-/-}$ mice are viable but female mice are unable to lactate [41]. 


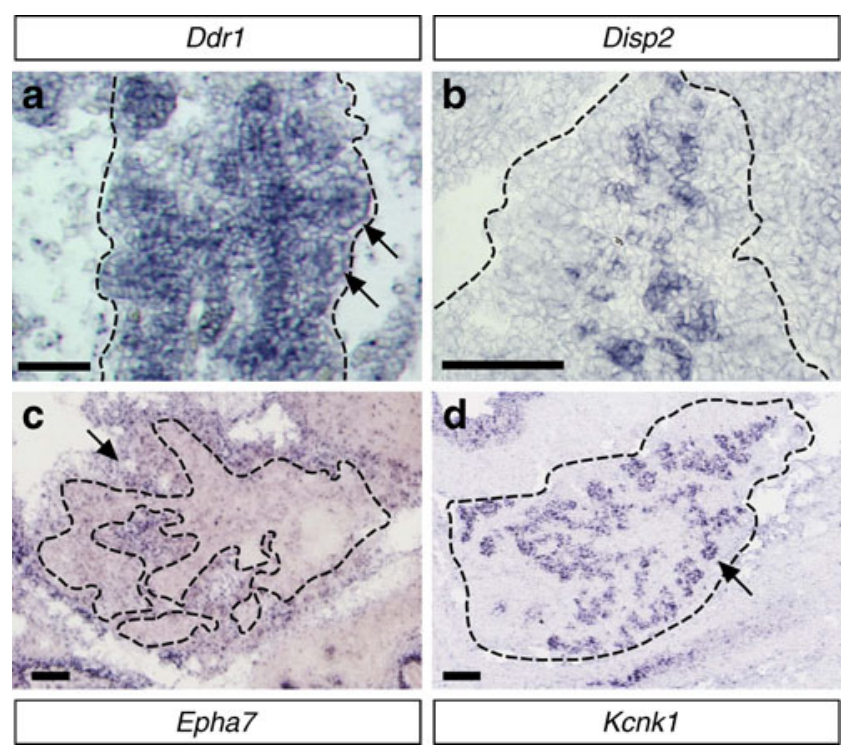

Fig. 2 Examples of categories of ISH expression patterns in the mouse pancreas. Embryonic mouse pancreatic endoderm is localised between (or within) the stippled lines. a $D d r 1$ has an 'Nkx6.1-like' expression in the central trunk-domain at day e15.5 (category 6). The central endoderm is positive for the ISH signal, whereas the acinar rosettes are negative, marked by arrows. b Disp2 is categorised as both 'Neurog3-like' (category 7) and 'Endocrine-like' (category 8) at day e15.5. c Epha7 is expressed in the mesenchyme (category 4) at day e14.5 (arrow). d A gene with acinar (category 5) expression pattern is Kcnkl. One acinar rosette is marked by the arrow. Scale bar is $100 \mu \mathrm{m}$

DNER (GeneID: 227325) is a single-span transmembrane Notch ligand, with 638 aa being extracellular. Dner ${ }^{-/}$mice are viable but display motor discoordination in the fixed bar and rota-rod tests [42]. DISP2 (GeneID: 214240) is a 12 transmembrane 1345 aa protein of which two-thirds are extracellular. Its function is unknown, but DISP1 is involved in release of bioactive hedgehog (cholesterolmodified) in mice [43, 44]. LRP11 (GeneID: 237253) is a 483 aa single-span transmembrane protein with 433 aa on the extracellular side. LRP proteins have a multitude of ligands and functions [45, 46]. SEZ6L2 (GeneID: 233878) is a 923 aa single-span transmembrane protein with 857 aa on the extracellular side. SEZ6L2 is known as a prognostic marker for lung cancer [47]. Sez6l2 ${ }^{-/}$mice show no particular abnormalities [48].

In addition, we confirmed previous published candidate surface markers for the mature islet cells including TSPAN7 [23], TMEM27 [24] (data not shown) and FXYD2 [19] (Fig. $5 \mathrm{~s}-\mathrm{u}$ ).

All markers identified have been targeted with antibodies directed towards predicted extracellular epitopes. Surface labelling is documented for all antibodies in the ESM (ESM Figs 2 and 3). In addition, these antibodies also displayed cytoplasmic staining (e.g. active protein biosynthesis, vesicular storage etc.) in IHC, which was useful to document the tissue-specific expression pattern of the different markers.

DDR1 is a bio-surface marker of central pancreatic endocrine and ductal progenitors IHC revealed DDR1 to be exclusively expressed in the endoderm (Fig. 3a-f) and in the progenitors of the central nervous system (data not shown) in e15.5 mouse embryos. In the endoderm DDR 1 is expressed in the lungs (data not shown), oesophagus (Fig. 3a) and in the upper stomach (cardia, fundus and body) (Fig. 3b). However, DDR1 is not expressed in the lower stomach (pyloric region) (Fig. 3c) or in the duodenum (Fig. 3f), but is expressed posterior to the duodenum in the gut tube (data not shown). In the posterior foregut area DDR 1 is highly pancreas-specific as observed by the sharp DDR1 expression boundary where the pancreas duct is fused with the duodenum (Fig. 3e). In the pancreas DDR1 is localised to the central 'trunk'domain known to harbour endocrine progenitors (Fig. 3d) and is highly co-expressed with NK6 homeobox 1 (NKX6.1) at this stage (Fig. 3g). Similarly, almost all NEUROG3-positive cells co-stain for DDR1 (Fig. 3h). It should be noted that many DDR1 cells are negative for NEUROG3. DDR1 is, therefore, not an exclusive marker of NEUROG3-positive cells but is a more a general marker of the trunk-domain similar to the transcription factor NKX6.1 at this stage. Expression of DDR1 starts around e11 in the mouse (data not shown) but is absent in the adult mouse and human pancreas (Fig. 3i, data not shown). To study DDR1 expression in fetal human pancreas corresponding to the stage of e15.5 mouse pancreas, we obtained embryonic human weeks of gestation (WG) 11 pancreas, which was further matured for 2 weeks in vitro. Under these conditions we observed that areas positive for NKX6.1 (Fig. 3j) also are positive for DDR1 (Fig. 3k). At high magnification it is evident that the co-expression of DDR1 and NKX6.1 is similar to that observed in mouse (Fig. 3n). Subsequently, we tested how DDR1 was expressed compared with PROM1, a biomarker reported to label the progenitors of the pancreas [22]. We find a prominent PROM1 staining lining the acinar rosettes, whereas only a closer inspection reveals a weak PROM $1^{+}$immunoreaction within the central NKX6.1/DDR1 double-positive domain that contains the NEUROG3-positive cells (Fig. 3o). In some acinar rosettes a weak DDR1 staining is found to be overlapping with PROM1 (Fig. 30). This is in agreement with NKX6.1 expression extending into the first part of some of the acinar rosettes at this time point (data not shown).

DNER is a bio-surface marker of pancreatic endocrine progenitors In the e15.5 mouse embryo endoderm DNER immunoreactivity is restricted to the pancreas. Outside the endoderm DNER expression is confined to neurons (data 


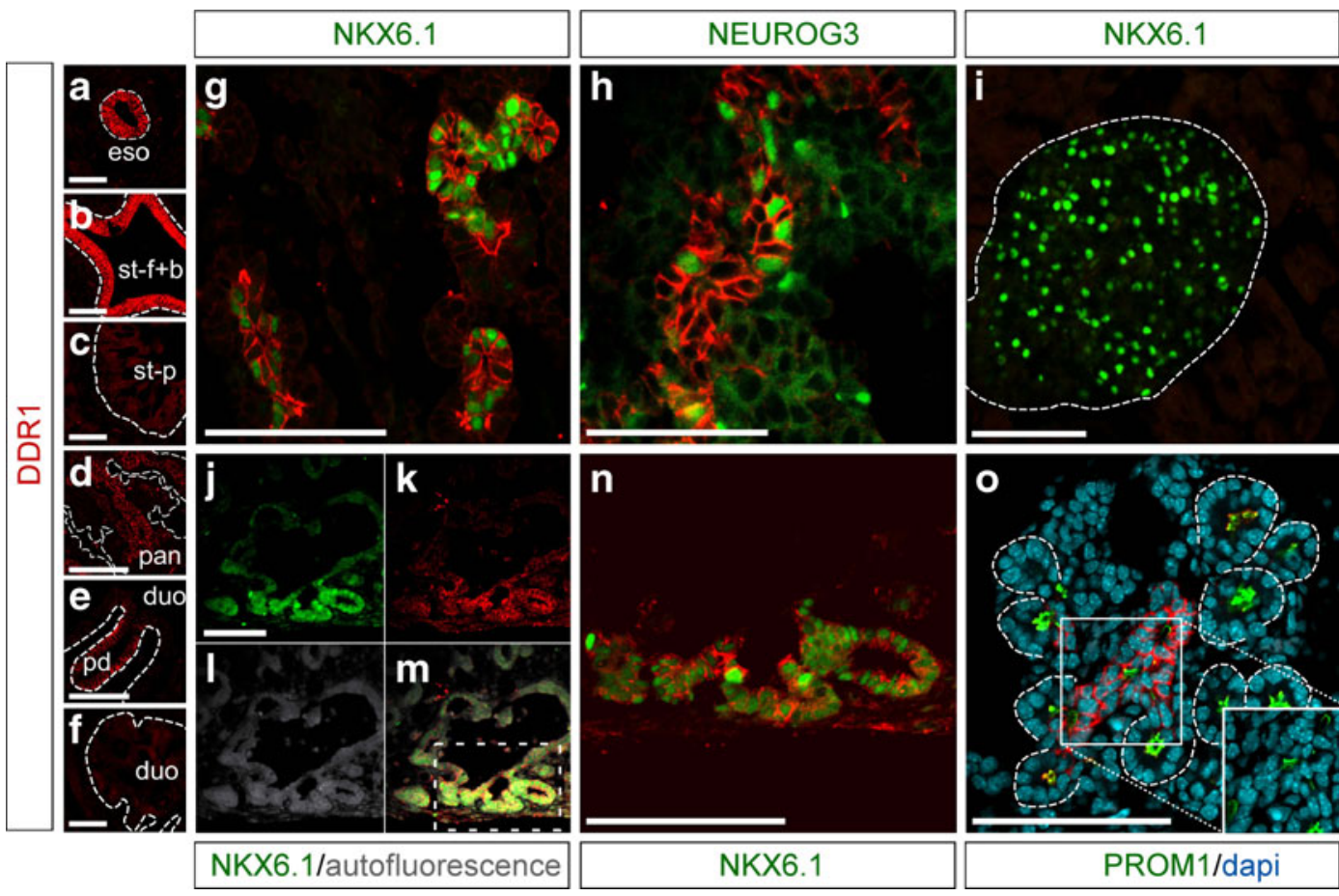

Fig 3 DDR1 marks pancreatic progenitors in mouse and human. DDR1 (red) is differentially expressed in the endoderm of e15.5 mouse embryo. Strong DDR1 expression is observed in the oesophagus (a) and the upper stomach (cardia, fundus and body) (b). However, DDR1 is not expressed in the lower stomach (pyloric region) of the stomach (c) nor in the duodenum (e and f). Interestingly, in the otherwise DDR1-negative posterior foregut, we observe a strong DDR1 expression in the central domain of the pancreas (d) and in the pancreas main duct resulting in a sharp DDR1 boundary at the pancreas/duodenum junction (e). The DDR1-positive (red) cells found in the central domain of the e 15.5 mouse are to a very large extent also positive for NKX6.1 (green) and nearly all NKX6.1-positive cells are DDR1-positive (g). NEUROG3-positive (green) cells are located in the central pancreas area, and we find close to all NEUROG3-positive cells also to be positive for DDR1 (red) (h). However, DDR1 (red) expression is not observed in the adult mouse pancreas or islet as marked by NKX6.1 (green) (islet is outlined with the stippled line) (i).

not shown). In the mouse pancreas DNER-positive cells are found as scattered cells in contrast to the continuous expression of DDR1. The DNER-positive cells are positive for PDX1 (Fig. 4a) and are found in the central pancreas domain (like DDR1) that give rise to the endocrine cells marked by high levels of PDX1, as observed on the whole mount projection (Fig. 4e). DNER is not co-expressed with PDX $1^{\text {high }}$ cells (Fig. 4a), which is consistent with insulin, glucagon and ghrelin-positive cells being negative for DNER (Fig. 4b, f and data not shown). DNER-positive cells are also negative for pancreas specific transcription factor, 1a (PTF1A) (data not shown) but a significant fraction of the DNER-positive cells co-express NEUROG3 (Fig. 4c). From the whole mount projection the similar 'salt and pepper' [49] distribution-pattern of DNER and NEUROG3-positive cells suggests a close and spatial
In human WG11 embryonic pancreas grown for 2 weeks on filter, cells expressing NKX6.1 (green) (j) co-express DDR1 (red) (k, m) in a pattern that appears to be identical to that observed in the embryonic mouse pancreas. All pancreatic cells are outlined with autofluorescence (grey) (l). At higher magnification (the stippled square in $\mathbf{m}$ ) the DDR1 (red) membrane staining around the NKX6.1-positive (green) nuclei is evident (n). Comparing DDR1 (red) with PROM1 (green) in the mouse pancreas, we find that strong PROM1 marks the acinar cells (outlined with stippled line and the DAPI-stained nuclei [blue]) and only weaker PROM1 expression marks the central pancreas domain where DDR1 immunoreactivity is found (o). Weak DDR1 immunoreactivity is found in some of the rosette cells next to the ducts that also express NKX6.1 (o and data not shown). Scale bar is $100 \mu \mathrm{m}$. duo, duodenum; eso, oesophagus; pan, pancreas; st-f $+\mathrm{b}$, fundus and body of the stomach; st-p, pyloric stomach. $\mathbf{j}-\mathbf{l}$ are single exposures of $\mathbf{m}$

relation of the cell populations (Fig. 4g). To establish the nature of DNER-positive cells that are negative for NEUROG3, we first stained for the post NEUROG3 marker PAX6. The majority of DNER-positive cells are PAX6-negative (Fig. 4d, arrowhead), but we do find few cells co-expressing DNER and PAX6 (Fig. 4d, arrow). Furthermore, the whole mount projection (Fig. 4h) shows PAX6 expression in clusters (endocrine cells) contrasting the single DNER-positive cells.

In cultured human WG11 embryonic pancreas, DNER immunoreactivity is mainly observed in single cells (Fig. 41) confirming what is observed in the mouse. DNER has a general weak islet-specific expression in the adult mouse pancreas but with a few strong DNER-positive islet cells that tend to co-express glucagon (Fig. 4i-k). In the human adult pancreas there is a strong patchy signal for 


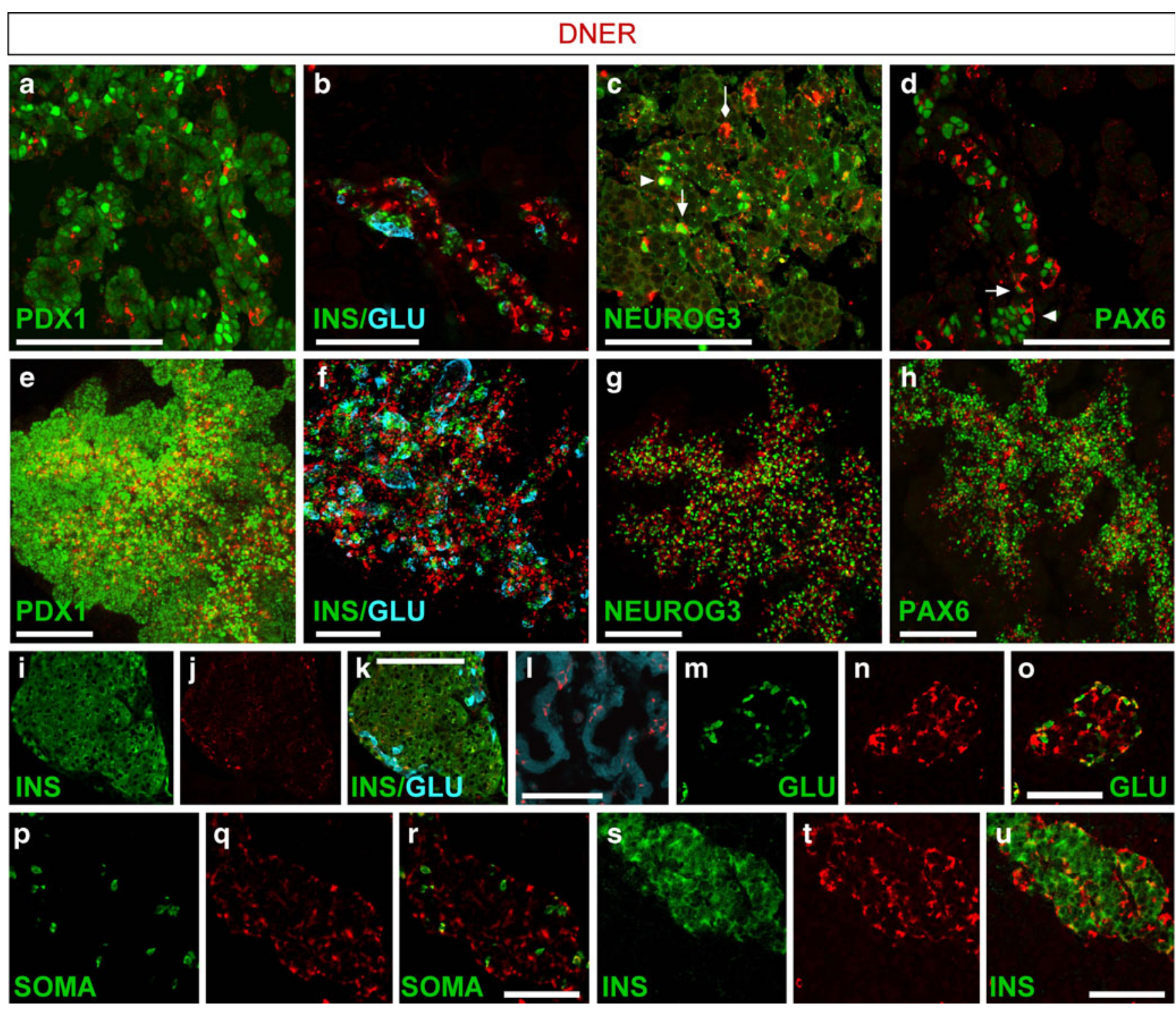

Fig. 4 DNER marks endocrine progenitors in the pancreas. At e15.5 DNER (red) is co-expressed with PDX1 (green) but absent from the PDX $1^{\text {high }}$ cells (a). Projection of optical sections from whole mount staining for DNER and PDX1 reveals that DNER is localised in the central trunk epithelium PDX1 ${ }^{\text {high }}$ area (e). DNER (red) is also absent from insulin (green) and glucagon-positive (blue) cells (b), but in close association with the endocrine clusters in the trunk epithelium (f). We find NEUROG3 (green) and DNER (red) to be co-expressed in about half of the DNER-positive cells. Arrow points to a NEU$\mathrm{ROG}^{+}{ }^{-} \mathrm{DNER}^{+}$, arrowhead points to a NEUROG3 ${ }^{+} / \mathrm{DNER}^{-}$cells and the diamond arrow points to a NEUROG3 ${ }^{-} / \mathrm{DNER}^{+}$. We consistently observe DNER to be asymmetrically localised in many cells (c). From the whole mount it is observed that DNER and NEUROG3 protein have the same 'salt and pepper'-like pattern (g). Co-staining of DNER (red) with PAX6 (green) reveals primarily two distinct cell populations (arrowhead: $\mathrm{DNER}^{+} / \mathrm{PAX}^{-}$cell), but co-

DNER in the islets. As in mouse, DNER overlaps partially with glucagon-positive cells (Fig. 4m-o), but interestingly also with somatostatin-positive cells (Fig. 4p-r). DNER is only very weakly co-expressed with insulin-positive cells (Fig. $4 \mathrm{~s}-\mathrm{u}$ ) as in the mouse. expression is detected in a minor fraction (arrow) (d). In projected whole mount stains it is observed that PAX6 is expressed more continuous and in patches contrasting the salt and pepper-like expression of DNER (h). In the adult mouse pancreas a weak panislet expression is observed for DNER (red) with a few cells in the periphery expressing DNER at a higher level of which a significant number co-express glucagon (blue) $(\mathbf{j}, \mathbf{k})$. Insulin is shown in green (i, k). In the human WG11 embryonic pancreas (differentiated further for 2 weeks on filter, in vitro) DNER (red) is also mainly expressed in a salt and pepper-like pattern (I). In the adult human pancreas DNER (red) and glucagon (green) are partially co-expressed as in the mouse (m-o). Some co-expression of DNER (red) with somatostatin (green) is also observed (p-r) but DNER (red) is not co-expressed with insulin (green) (s-u). $\mathbf{i}, \mathbf{m}, \mathbf{p}$ and $\mathbf{s}$ are single channel (green) exposure of $\mathbf{k}, \mathbf{o}, \mathbf{r}$ and $\mathbf{u}$ respectively. Scale bar is $100 \mu \mathrm{m}$

SEZ6L2, DISP2 and LRP11 are bio-surface markers of hormone-positive cells IHC staining with anti-SEZ6L2 and anti-LRP11 reveal an expression pattern within the endoderm to be specific for pancreatic islet and gut endocrine cells. Outside the endoderm SEZ6L2 and LRP11 stain 
neurons (data not shown). In the pancreas SEZ6L2 and LRP11 co-localise to all endocrine (hormone-containing) cells starting from e10.5 in the mouse. Also at e11.5 and e12.5 SEZ6L2 and LRP11 co-localise with insulin and glucagon (Fig. 5a-f, only SEZ6L2 is shown as SEZ6L2 and LRP11 stain identical cells). From around e13.5, SEZ6L2 also marks a few NKX6.1 ${ }^{+} / \mathrm{Ins}^{-}$(data not shown). At e18.5 SEZ6L2 continues to be expressed in insulin, glucagon and ghrelin-positive cells (Fig. 5g-i, and data not shown). In the adult human and mouse pancreas SEZ6L2 (Fig. 5j-1 and data not shown), DISP2 (Fig. 5m-o, data not shown) and LRP11 (Fig. 5p-r, and data not shown) all stain the islet. On mouse and human tissue the DISP2 staining is localised to the islet. Endocrine-specificity of Lrp11, Sez6l2 and Disp2 was validated by lack of signal (ISH and IHC) on e15.5 Neurog3 null mouse tissue (data not shown). The published beta cell specific marker FXYD2 $\gamma$ [19] marks the mouse islet as reported (Fig. $5 \mathrm{~s}-\mathrm{u}$ ).

DNER and DDR1 are upregulated in PDL pancreas Since we defined DDR1 and DNER as fetal endocrine pancreas progenitor markers we tested for an upregulation/reexpression in the adult pancreas that was injured by PDL to activate beta cell progenitors [4]. The PDL pancreas displays a strong continuous expression of DDR1 immunoreactivity in the PDX1-positive progenitor area (ESM Fig. 1h) reminiscent of the e15.5 embryonic mouse pancreas and in the human embryonic pancreas (Fig. 3). Strong upregulation of DNER is observed in the islets (ESM Fig. 1n) and a weaker expression in a 'salt and pepper' pattern is evident in the PDX1 progenitor area (ESM Fig. 1m-0). This expression pattern corresponds to what is observed in the embryonic mouse and human pancreas.

Progenitor and mature cell markers can be used to sort cells Having established expression patterns of the biosurface markers in mouse, man and in a regeneration model, we next set out to see if the markers can be used for sorting cells. In agreement with our IHC data we found alphaTC cells to be positive for DISP2 (Fig. 6a). Preparing single alphaTC cells by using cell dissociation buffer and staining with our monoclonal antibody DISP2 results in a clear shift (Fig. 6b, red line) compared with the mouse isotype control antibody (Fig. 6b, black line). The DISP2 antibody also works in FACS on cells that have been dissociated with trypsin (data not shown). AlphaTC is also positive for DNER and negative for DDR1, in agreement
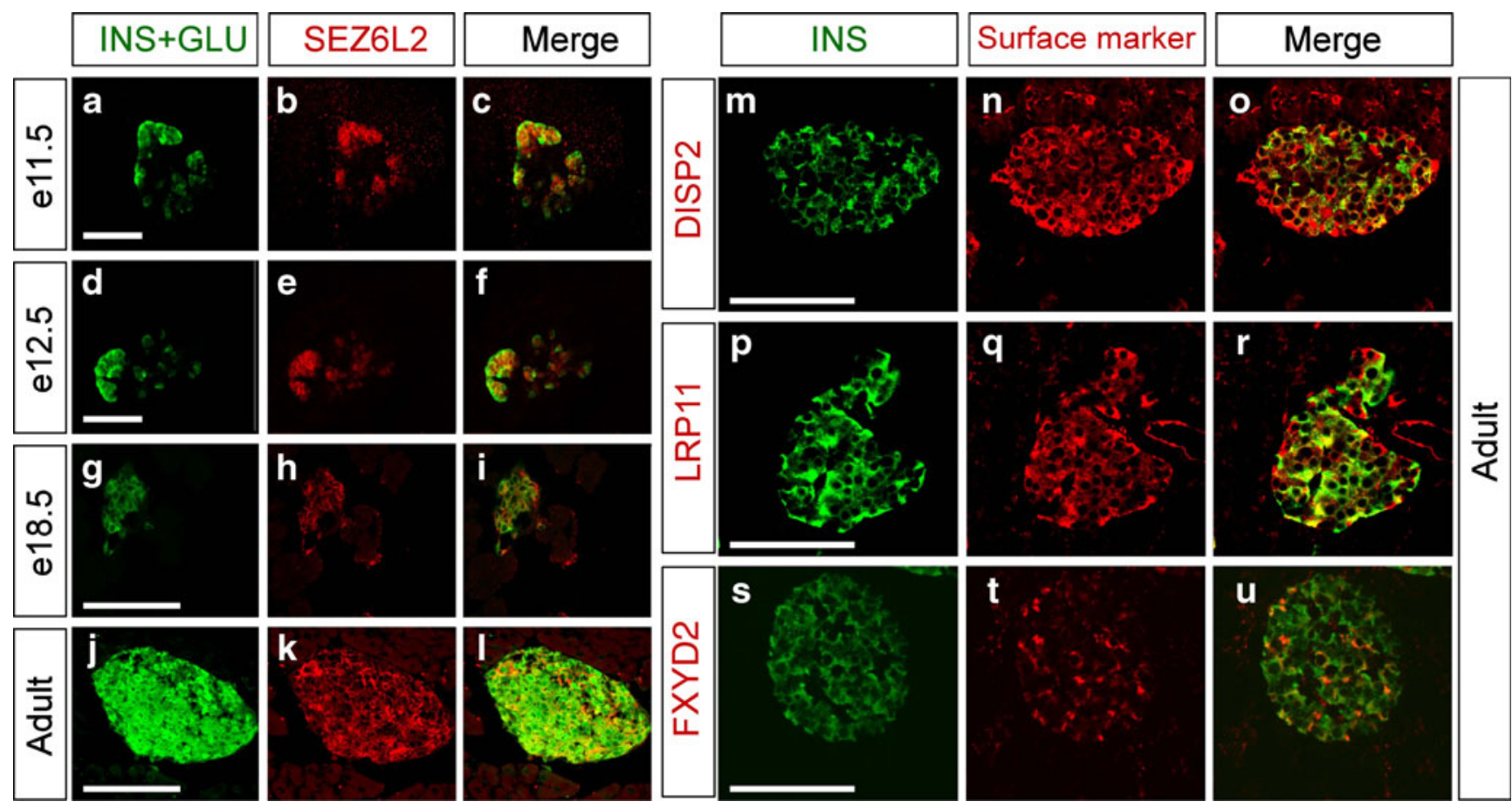

Fig. 5 Pancreas expression of mature islet cell bio-markers: SEZ6L2, DISP2, LRP11 and FXYD2. Robust expression of SEZ6L2 (red) can be detected in glucagon- and insulin-positive (green) cells in the mouse embryo at e11.5 (a-c) and e12.5 (d-f). At e18.5 SEZ6L2 (red) remains expressed in the insulin and glucagon (green) hormonepositive cells (g-i). In the adult mouse (and human, data not shown) pancreas SEZ6L2 (red) continues to mark insulin and glucagon- positive (green) cells $(\mathbf{j}-\mathbf{k})$. In the adult human pancreas DISP2 (red) stains the islet cells shown with insulin (green) (m-0). LRP11 appears to be expressed like SEZ6L2 throughout mouse development. In the adult human pancreas LRP11 (red) marks the islet, here highlighted with insulin (green) (p-r). Staining for FXYD2 (red) on adult mouse pancreas shows a patchy islet expression. Islet is marked with insulin (green) (s-u). Scale bar is $100 \mu \mathrm{m}$ 

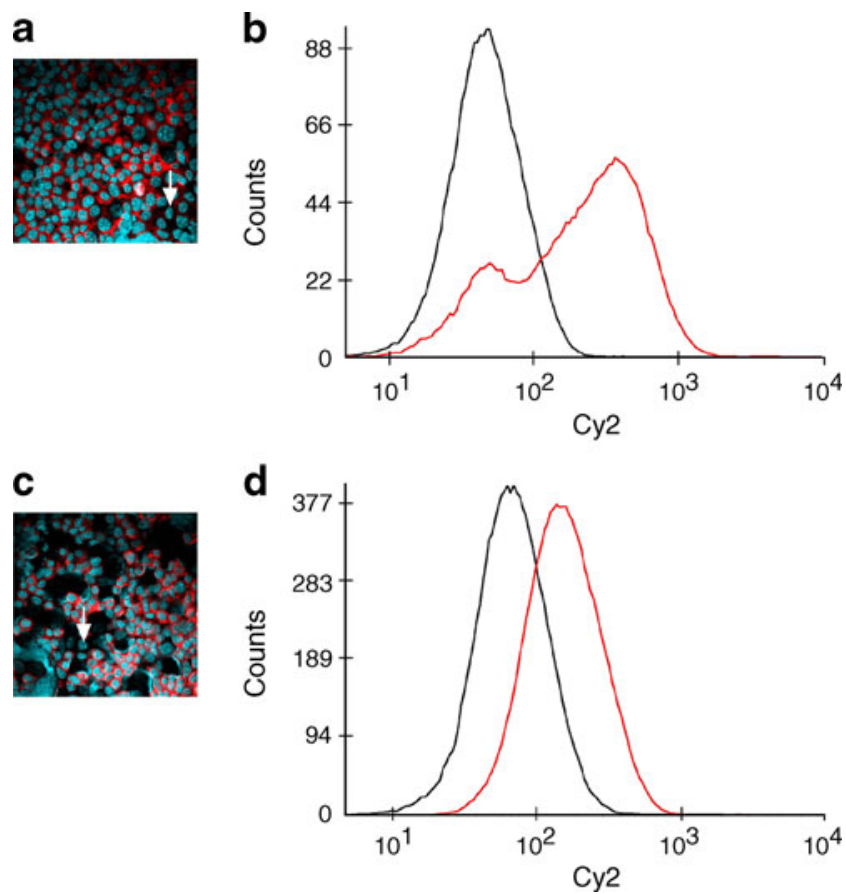

Fig. 6 DISP2 and DNER work in cell sorting applications. Performing cytospin of dissociated AlphaTC cells allows immunocytochemical detection of DISP2 by using monoclonal anti-DISP2 antibody, F66 (a). This cell line is not homogeneously positive for DISP2 (negative cell shown by arrow). FACS analysis of single alphaTC cells stained for DISP2 show a marked shift in fluorescence (b, red line) compared with the control $\operatorname{IgG}_{1}$-stained cells (b, black line). Note that some of the alphaTC cells are not shifted, reflecting the DISP2-negative cell population observed in (a). AlphaTC also express immunoreactive DNER (c) and can be shifted by FACS following staining with anti DNER polyclonal $\mathrm{IgG}$ fraction (d, red line) compared with alphaTC stained with control IgG (d, black line)

with our IHC data (Fig. 6c and data not shown). Polyclonal anti-DNER reagent of goat origin was used in FACS application and compared with non-immune goat IgG. Again anti-DNER is able to shift the alphaTC population (Fig. 6d red line) compared with the control IgG (Fig. 6d, black line).

\section{Discussion}

Using a comprehensive bioinformatics approach combined with ISH and IHC screens we present an accumulating panel of surface marker antigens. We show that these are expressed during pancreatic development at various levels of endocrine cell maturation: either before endocrine specification (DDR1), around endocrine cell specification (DNER) or after the progenitor state (DISP2, LRP11, SEZ6L2). These markers therefore provide novel candidates for future purification of cells at various stages of endocrine maturation.
We find DDR1 to specifically label the centralised field of multipotent pancreatic progenitors at the secondary transition from which NEUROG3-positive cells emerge. The DDR1-positive cells that are NEUROG3-negative are likely to contain the pre-NEUROG3 cell as well as ductal committed cells. The DDR1-positive cell population also contain proliferating cells (data not shown). Strikingly, the DDR1 expression area in the ductal-trunk domain sharply demarks the border between the pancreatic ductal junctions to the duodenal epithelium. Within the early duodenal domain the DDR1-positive cells thus mark the committed pancreatic progenitor cells that not only harbour the endocrine cell differentiation potential but also cells with a proliferation potential. Similar cells could potentially be isolated from the hESC culture and expanded before final differentiation.

Of interest to the dynamics within the central trunk-domain is the DNER-labelling of non-dividing (data not shown) cell populations partially overlapping with $\mathrm{NEUROG}^{+}$cells but also cells that are hormone ${ }^{-} / \mathrm{NEUROG}^{-}$. DNER does not follow any known marker and we therefore speculate that DNER marks cells prior to, as well as at, the early NEUROG3-expressing stage. Furthermore, DNER is exclusively expressed in the pancreas within the endoderm making it a very attractive surface marker for the pancreatic endocrine progenitor cell pool at the NEUROG3-stage. The progenitor nature of DDR1- and DNER-expression was also confirmed in the PDL model where we observed a reexpression of DDR1 in the regenerating tissue and an upregulation of DNER in the pre-existing islets. In the human system, the DDR1 expression recapitulates that of the mouse, making DDR1 an attractive surface tag for isolating both mouse and human pancreatic progenitor cells from mixed populations. DNER is expressed in the human embryonic pancreas in a pattern that also mirrors that observed in the mouse. In the adult human pancreas DNER tends to co-localise with the glucagon-positive cells, but also with other islet cell types albeit at lower expression level.

Compared with the published PROM1 surface marker that has also been proposed to be of use in purification/ isolation, we find that PROM1 has low expression levels in the DDR1/DNER central pancreas area when compared with its expression levels in the acinar cells. We have now shown that a polyclonal antiserum to DNER also works in FACS, thus providing a potential useful tool to purify endocrine progenitor cells from fetal pancreatic tissue as well as from stem cell-derived cultures. So far, commercially available monoclonal DNER-antibodies have not worked in FACS purification but future monoclonal antibodies may recognise available surface epitopes to serve such a purpose.

We characterised DISP2, SEZ6L2 and LRP11 as panendocrine islet markers. Peptide designs (to optimise for 
human cross-reaction) from all three proteins representing putative extracellular accessible epitopes were successfully used in generating polyclonal as well as monoclonal (DISP2) antibodies. This was demonstrated by our FACS data, with DISP2 resulting in a clean separation between DISP2-positive and -negative cells. The DISP2 monoclonal antibody F66 fully cross-reacted to human DISP2 as verified by IHC. Interestingly, our DISP2 antibody works equally well in FACS after use of trypsin compared with cell dissociation buffer. We predict the three surface markers to be useful in the purification and/or imaging of hormonepositive islet cells. Our list of membrane proteins also contained TSPAN7 and TMEM27 [23, 24] recently identified by others but, for example FXYD2 [19] was not on any of our three lists, thus predicting that additional beta cell- or progenitor-specific surface markers are yet to be identified.

In summary, we have identified novel surface markers for pancreatic endocrine progenitors as well as of mature endocrine cells. Antibodies to such extracellular epitopes could facilitate cell purification steps in protocols involving differentiation of pluripotent stem cells towards glucosesensitive beta cells for diabetes therapy. The described approach is based on bioinformatics leading to identification of putative candidate antigens that can result in useful antibody generation. This approach complements a recently published strategy using whole cellular immunisation to identify monoclonal antibodies against cell stage-specific epitopes [13, 28], which together have resulted in a panel of novel immunoisolation tools that will prove to be instrumental to further characterise and dissect the successive differentiation states characterising the formation of mature insulin-producing beta cells.

Acknowledgements This work was supported by the National Institute of Diabetes \& Kidney Diseases of the National Institutes of Health, Bethesda, MD (U01DK072473) as part of the Antibody Core of the Beta Cell Biology Consortium. J. Hald was supported by 'MADBETA-Grant' (U01DK072473). O.D. Madsen was supported by the European Union 6th Framework Programme (BetaCellTherapy, 512145) as part of the Juvenile Diabetes Research Foundation Center for Beta Cell Therapy of Diabetes. The authors thank S. Refsgaard Lindskog, A. Bjerregaard, M. Lauritzen and G. Leuckx for expert technical assistance and P. Serup for the interpretation of DDR1 data sets. R Gorski (Gorski Consulting, Philadelphia, PA, USA) contributed to the unbiased EPCon gene list

Contribution statement $\mathrm{JH}$ planned and designed the experiments, obtained and analysed the data, performed the experiment in Fig. 6, drew the figures and wrote the paper. TG carried out the experimental analysis and interpretation of data leading to Fig. 5. CR did the experiment on FXYD2 and captured and interpreted the data. LR and AES designed and carried out the experimental analysis leading to the production of the DISP2 monoclonal antibody as well as the polyclonal antisera to LRP11 and SEZ6L2 and participated in their characterisation. HH designed the PDL experiment including interpretation of data. JA-R did the whole mount stainings and acquired/analysed the whole mount data in Fig. 4. JJ provided the Neurog3 knockout array data including thorough bioinformatic input and prioritization of candidates, and contributed to the generation of Fig. 1. RS designed experiments and provided the embryonic human tissue and participated in evaluating human antigen expression. GG participated in the design of overall strategy and experiments and provided the Neurog3-YFP array data and participated in the ISH screen. CS Jr and KK participated in the original conceptual design of the experimental approach, provided the unbiased EPCon gene list and participated in analysis and interpretation of data. JNJ gave critical input and focused the experiments. ODM conceived the studies and provided critical input in data analysis and interpretation and co-wrote the paper. All authors have read and given critical input during revisions of the paper and all have approved the final version.

Duality of interest G. Gradwohl, J. Hald and O.D. Madsen have filed a patent on the use of DNER as a surface tag for immunoisolation. J. Hald and O.D. Madsen have filed a patent on the use of DDR1 as a surface tag for immunoisolation. The remaining authors declare that there is no duality of interest associated with this manuscript.

\section{References}

1. Butler AE, Janson J, Bonner-Weir S, Ritzel R, Rizza RA, Butler PC (2003) Beta-cell deficit and increased beta-cell apoptosis in humans with type 2 diabetes. Diabetes 52:102-110

2. Rahier J, Guiot Y, Goebbels RM, Sempoux C, Henquin JC (2008) Pancreatic beta-cell mass in European subjects with type 2 diabetes. Diabetes Obes Metab 10(Suppl 4):32-42

3. Madsen OD (2005) Stem cells and diabetes treatment. APMIS 113:858-875

4. Xu X, D'Hoker J, Stange G et al (2008) Beta cells can be generated from endogenous progenitors in injured adult mouse pancreas. Cell 132:197-207

5. Shapiro AM, Lakey JR, Ryan EA et al (2000) Islet transplantation in seven patients with type 1 diabetes mellitus using a glucocorticoid-free immunosuppressive regimen. N Engl J Med 343:230-238

6. Shapiro AM, Ricordi C, Hering BJ et al (2006) International trial of the Edmonton protocol for islet transplantation. N Engl J Med 355:1318-1330

7. Kroon E, Martinson LA, Kadoya K et al (2008) Pancreatic endoderm derived from human embryonic stem cells generates glucose-responsive insulin-secreting cells in vivo. Nat Biotechnol 26:443-452

8. D'Amour KA, Bang AG, Eliazer S et al (2006) Production of pancreatic hormone-expressing endocrine cells from human embryonic stem cells. Nat Biotechnol 24:1392-1401

9. Jiang J, Au M, Lu K et al (2007) Generation of insulin-producing islet-like clusters from human embryonic stem cells. Stem Cells 25:1940-1953

10. Zhang D, Jiang W, Liu M et al (2009) Highly efficient differentiation of human ES cells and iPS cells into mature pancreatic insulin-producing cells. Cell Res 19:429-438

11. Tateishi K, He J, Taranova O, Liang G, D'Alessio AC, Zhang Y (2008) Generation of insulin-secreting islet-like clusters from human skin fibroblasts. J Biol Chem 283:31601-31607

12. Challen GA, Boles N, Lin KK, Goodell MA (2009) Mouse hematopoietic stem cell identification and analysis. Cytometry A $75: 14-24$

13. Dorrell C, Abraham SL, Lanxon-Cookson KM, Canaday PS, Streeter PR, Grompe M (2008) Isolation of major pancreatic cell 
types and long-term culture-initiating cells using novel human surface markers. Stem Cell Res 1:183-194

14. Contreas G, Jorgensen J, Madsen OD (1990) Novel islet, duct, and acinar cell markers defined by monoclonal autoantibodies from prediabetic BB rats. Pancreas 5:540-547

15. Brogren CH, Hirsch F, Wood P, Druet P, Poussier P (1986) Production and characterization of a monoclonal islet cell surface autoantibody from the BB rat. Diabetologia 29:330-333

16. Ueberberg S, Ziegler D, Schechinger W et al (2010) In vitro phage display in a rat beta cell line: a simple approach for the generation of a single-chain antibody targeting a novel beta cell-specific epitope. Diabetologia 53:1384-1394

17. Ueberberg S, Schneider S (2010) Phage library-screening: a powerful approach for generation of targeting-agents specific for normal pancreatic islet-cells and islet-cell carcinoma in vivo. Regul Pept 160:1-8

18. Ueberberg S, Meier JJ, Waengler C et al (2009) Generation of novel single-chain antibodies by phage-display technology to direct imaging agents highly selective to pancreatic beta- or alphacells in vivo. Diabetes 58:2324-2334

19. Flamez D, Roland I, Berton A et al (2010) A genomic-based approach identifies FXYD domain containing ion transport regulator 2 (FXYD2)gammaa as a pancreatic beta cell-specific biomarker. Diabetologia 53:1372-1383

20. Gouon-Evans V, Boussemart L, Gadue P et al (2006) BMP-4 is required for hepatic specification of mouse embryonic stem cellderived definitive endoderm. Nat Biotechnol 24:1402-1411

21. Cai J, Yu C, Liu Y et al (2010) Generation of homogeneous PDX1 $(+)$ pancreatic progenitors from human ES cell-derived endoderm cells. J Mol Cell Biol 2:50-60

22. Sugiyama T, Rodriguez RT, McLean GW, Kim SK (2007) Conserved markers of fetal pancreatic epithelium permit prospective isolation of islet progenitor cells by FACS. Proc Natl Acad Sci U S A 104:175-180

23. Lindskog C, Asplund A, Engkvist M, Uhlen M, Korsgren O, Ponten F (2010) Antibody-based proteomics for discovery and exploration of proteins expressed in pancreatic islets. Discov Med 9:565-578

24. Altirriba J, Gasa R, Casas S et al (2010) The role of transmembrane protein 27 (TMEM27) in islet physiology and its potential use as a beta cell mass biomarker. Diabetologia 53:1406-1414

25. Saisho K, Fukuhara A, Yasuda T et al (2009) Glucose enhances collectrin protein expression in insulin-producing MIN6 beta cells. Biochem Biophys Res Commun 389:133-137

26. Malakauskas SM, Kourany WM, Zhang XY et al (2009) Increased insulin sensitivity in mice lacking collectrin, a downstream target of HNF-1alpha. Mol Endocrinol 23:881-892

27. Dreja T, Jovanovic Z, Rasche A et al (2010) Diet-induced gene expression of isolated pancreatic islets from a polygenic mouse model of the metabolic syndrome. Diabetologia 53:309-320

28. Gadue P, Gouon-Evans V, Cheng X et al (2009) Generation of monoclonal antibodies specific for cell surface molecules expressed on early mouse endoderm. Stem Cells 27:2103-2113

29. Juhl K, Sarkar SA, Wong R, Jensen J, Hutton JC (2008) Mouse pancreatic endocrine cell transcriptome defined in the embryonic Ngn3-null mouse. Diabetes 57:2755-2761

30. Soyer J, Flasse L, Raffelsberger W et al (2010) Rfx6 is an Ngn3dependent winged helix transcription factor required for pancreatic islet cell development. Development 137:203-212

31. Ahnfelt-Ronne J, Hald J, Bodker A, Yassin H, Serup P, HecksherSorensen J (2007) Preservation of proliferating pancreatic pro- genitor cells by Delta-Notch signaling in the embryonic chicken pancreas. BMC Dev Biol 7:63

32. Pedersen IL, Klinck R, Hecksher-Sorensen J et al (2006) Generation and characterization of monoclonal antibodies against the transcription factor Nkx6.1. J Histochem Cytochem 54:567574

33. Ahnfelt-Ronne J, Jorgensen MC, Hald J, Madsen OD, Serup P, Hecksher-Sorensen J (2007) An improved method for threedimensional reconstruction of protein expression patterns in intact mouse and chicken embryos and organs. J Histochem Cytochem 55:925-930

34. Castaing M, Peault B, Basmaciogullari A, Casal I, Czernichow P, Scharfmann R (2001) Blood glucose normalization upon transplantation of human embryonic pancreas into beta-cell-deficient SCID mice. Diabetologia 44:2066-2076

35. Castaing M, Duvillie B, Quemeneur E, Basmaciogullari A, Scharfmann R (2005) Ex vivo analysis of acinar and endocrine cell development in the human embryonic pancreas. Dev Dyn 234:339-345

36. Attali M, Stetsyuk V, Basmaciogullari A et al (2007) Control of beta-cell differentiation by the pancreatic mesenchyme. Diabetes 56:1248-1258

37. Haumaitre C, Lenoir O, Scharfmann R (2008) Histone deacetylase inhibitors modify pancreatic cell fate determination and amplify endocrine progenitors. Mol Cell Biol 28:6373-6383

38. Mellitzer G, Martin M, Sidhoum-Jenny M et al (2004) Pancreatic islet progenitor cells in neurogenin 3-yellow fluorescent protein knock-add-on mice. Mol Endocrinol 18:2765-2776

39. Vogel W (1999) Discoidin domain receptors: structural relations and functional implications. FASEB J 13(Suppl):S77-82

40. Vogel WF (2002) Ligand-induced shedding of discoidin domain receptor 1. FEBS Lett 514:175-180

41. Vogel WF, Aszodi A, Alves F, Pawson T (2001) Discoidin domain receptor 1 tyrosine kinase has an essential role in mammary gland development. Mol Cell Biol 21:2906-2917

42. Tohgo A, Eiraku M, Miyazaki T et al (2006) Impaired cerebellar functions in mutant mice lacking DNER. Mol Cell Neurosci $31: 326-333$

43. Burke R, Nellen D, Bellotto M et al (1999) Dispatched, a novel sterol-sensing domain protein dedicated to the release of cholesterol-modified hedgehog from signaling cells. Cell 99:803-815

44. Ma Y, Erkner A, Gong R et al (2002) Hedgehog-mediated patterning of the mammalian embryo requires transporter-like function of dispatched. Cell 111:63-75

45. Lillis AP, Mikhailenko I, Strickland DK (2005) Beyond endocytosis: LRP function in cell migration, proliferation and vascular permeability. J Thromb Haemost 3:1884-1893

46. May P, Woldt E, Matz RL, Boucher P (2007) The LDL receptorrelated protein (LRP) family: an old family of proteins with new physiological functions. Ann Med 39:219-228

47. Ishikawa N, Daigo Y, Takano A et al (2006) Characterization of SEZ6L2 cell-surface protein as a novel prognostic marker for lung cancer. Cancer Sci 97:737-745

48. Miyazaki T, Hashimoto K, Uda A et al (2006) Disturbance of cerebellar synaptic maturation in mutant mice lacking BSRPs, a novel brain-specific receptor-like protein family. FEBS Lett 580:4057-4064

49. Kageyama R, Ohtsuka T, Shimojo H, Imayoshi I (2008) Dynamic Notch signaling in neural progenitor cells and a revised view of lateral inhibition. Nat Neurosci 11:1247-1251 